\title{
Multiplex array proteomics detects increased MMP-8 in CSF after spinal cord injury
}

\author{
Matthew Light ${ }^{1}$, Kenneth H Minor ${ }^{1}$, Peter DeWitt ${ }^{2}$, Kyle H Jasper ${ }^{1}$ and Stephen JA Davies ${ }^{1 *}$
}

\begin{abstract}
Introduction: A variety of methods have been used to study inflammatory changes in the acutely injured spinal cord. Recently novel multiplex assays have been used in an attempt to overcome limitations in numbers of available targets studied in a single experiment. Other technical challenges in developing pre-clinical rodent models to investigate biomarkers in cerebrospinal fluid (CSF) include relatively small volumes of sample and low concentrations of target proteins. The primary objective of this study was to characterize the inflammatory profile present in CSF at a subacute time point in a clinically relevant rodent model of traumatic spinal cord injury (SCI). Our other aim was to test a microarray proteomics platform specifically for this application.

Methods: A 34 cytokine sandwich ELISA microarray was used to study inflammatory changes in CSF samples taken 12 days post-cervical $\mathrm{SCl}$ in adult rats. The difference between the median foreground signal and the median background signal was measured. Bonferroni and Benjamini-Hochburg multiple testing corrections were applied to limit the False Discovery Rate (FDR), and a linear mixed model was used to account for repeated measures in the array.
\end{abstract}

Results: We report a novel subacute SCI biomarker, elevated levels of matrix metalloproteinase-8 protein in CSF, and discuss application of statistical models designed for multiplex testing.

Conclusions: Major advantages of this assay over conventional methods include high-throughput format, good sensitivity, and reduced sample consumption. This method can be useful for creating comprehensive inflammatory profiles, and biomarkers can be used in the clinic to assess injury severity and to objectively grade response to therapy.

Keywords: biomarker, cerebrospinal fluid (CSF), cytokine, inflammation, matrix metalloproteinase-8 (MMP-8), microarray, proteomics, spinal cord injury, tissue inhibitor of metalloproteinase-1 (TIMP-1).

\section{Introduction}

Traumatic injury to the spinal cord can result in lifechanging neurological deficits. Patients, family members, clinicians, and researchers alike face significant longterm challenges including (but not limited to) pain management, rehabilitation, and functional improvement. According to 2011 estimates, the incidence of spinal cord injury (SCI) is roughly 12,000 cases per year in the USA, $40 \%$ resulting from motor vehicle accidents and $27 \%$ result from falls, creating a bimodal age distribution in

\footnotetext{
* Correspondence: Stephen.Davies@UCDenver.edu

'Department of Neurosurgery, University of Colorado School of Medicine, Building RC-1 North, Room P18-9400, 12800 E 19th Avenue, Aurora, CO 80045, USA

Full list of author information is available at the end of the article
}

the patient population [1]. Long-term implications of these debilitating injuries are significant. Advances in patient care, specifically prevention and treatment of urinary tract complications and renal failure, have improved longevity such that causes of mortality are now similar to the general population (cardiovascular disease, cancer, and lower respiratory disease) $[2,3]$.

The molecular mechanisms underlying inflammationinduced damage and recovery of function at the acute, subacute and chronic stages after SCI are currently being pieced together. Although trauma to the spinal cord results in rapid loss of neurons and glia within gray and white matter and transection of white matter pathways, this acute insult also initiates a robust inflammatory response [4-6] that continues to damage tissue 
$[7,8]$, creating new deficits at subacute and chronic time points post-injury. Loss of blood-spinal cord barrier (BSCB) [9] and lymphocytic infiltrate into the injury site [10] potentiates inflammation, and reactive gliosis and formation of scar tissue prevent axon regeneration $[5,11,12]$.

With clinical trials testing the effects of new therapies on neurologic outcomes post-SCI already underway (minocycline and erythropoetin to name a few) [13], and more still in the scientific pipeline, the role of cerebrospinal fluid (CSF) biomarkers as indicators of injury progression and repair is now more important than ever. Although functional improvement remains the gold standard and certainly the most important outcome for patients, Kwon et al. highlight the potential role for biomarkers in assessing initial injury severity and tracking changes in injury status over time. In addition, they emphasize the role of biomarkers in determining therapeutic response more objectively than current highly subjective functional outcome measures like the American Spinal Injury Association (ASIA) Score [14]. Other important potential applications include characterization of molecular signs of neurotoxic damage and endogenous neuro-repair, and discovery of new therapeutic targets. What is needed is a protein-based high-throughput assay that can detect biomarkers from a safe and clinically available source. Sensitivity is also critical because CSF (an important source of biomarkers given its proximity to neuronal tissue) contains relatively low protein concentrations even after BSCB disruption [15]. This ideal assay would be of significant value to researchers and clinicians alike, facilitating delivery of new therapies to SCI patients.

It is becoming increasingly apparent that inflammation plays key roles in both acute and secondary damage, and late-phase recovery after traumatic SCI, and that any novel therapy would almost certainly interact with these processes. In fact, much can be learned from the Immunology field, where researchers have adopted many of the latest technologies to characterize inflammatory processes previously too complex for traditional methods of investigation. Where PCR and standard ELISA were once popular for detecting changes in mRNA and protein respectively, newer multiplex arrays have emerged as the current assays of choice. Recently Lee $e t$ al. simultaneously profiled 10 different cytokine responses to H1N1 infection in hospitalized patients using a cytometric bead array system [16], and Mizutani et al. were able to track serial changes in 17 cytokines using a similar suspension array in patients with Crohn's disease treated with Infliximab [17]. Oncology is another field that has made good on the promise of newer array technology. Tefferi et al. studied the prognostic value of 30 cytokines in primary myelofibrosis by multiplex sandwich immunoassay [18]: one of many truly comprehensive cytokine profiling studies. For a thorough review of multiplex sandwich ELISA technology read Nielsen and Geierstanger [19].

An effort has also been made to validate the reliability of these new assays, focusing mainly on the issues surrounding transition from a uniplex to multiplex format. Pang et al. studied two commercially available solutionphase microarray platforms against standard ELISA in detecting autoantibodies in human serum [20]. They note similar detection performance, and name highthroughput screening and reduced reagent consumption as major advantages over standard methods. This paper also emphasizes the importance of cutoff values (criteria for determining positive and negative signals in the dataset). In further considering data processing, the multiplex format necessitates different statistical treatment of the data: the need for multiple hypothesis testing. The concept of the false discovery rate (FDR) comes from earlier work in epidemiology and genomewide association studies: the idea that as the number of statistical tests performed increases, so does the number of false positives. Hsueh et al. compare strategies like Benjamini-Hochburg [21] for estimating the FDR, and emphasize the importance of controlling the type I error rate in multiplicity testing [22]. Finally, important technical issues such as binding 'inhomogeneity' across immobilized spot-based arrays [23] are currently under investigation. To summarize, multiplex proteomic assays, although relatively new, offer many qualities of the 'ideal assay' described above, provided there is: correct statistical treatment of the data, careful consideration of technical issues, and verification of 'hits' with appropriate confirmatory methods.

The challenge of characterizing the inflammatory response to acute $\mathrm{SCI}$ has been met using a variety of methods from flow cytometry, to PCR, to ELISA. Streit et al. showed transient increases in IL-1 $\beta$, TNF- $\alpha$, IL-6, and M-CSF mRNA by PCR from cord tissue in a rat model of SCI (C8 contusion) [24]. Wang et al. also showed elevated IL-1 $\beta$ mRNA in contused cord, but extended their analysis by showing elevated protein levels in cord by ELISA. Of note, CSF samples also contained low levels of IL-1 $\beta$ [25]. These studies, and others, established the presence of inflammatory molecules in the setting of acute SCI, and yet they were clearly limited by the number of molecules that could be studied. More recently, the emergence of highthroughput multiplex techniques has allowed for more comprehensive analyses. Kwon et al. studied 25 cytokines (as well as a number of growth factors) simultaneously in human CSF samples from acute SCI patients [26]. Lubieniecka et al. identified 42 possible biomarkers (cytokines, growth factors, etc.) in CSF from a rat model 
24 hours post-injury by mass spectrometry of which 10 correlated with injury severity (ASIA Score) including Ywhaz, Itih4, and Gpx3 [27].

In an effort to characterize inflammatory markers present at subacute time points after traumatic SCI, thought to represent a clinically relevant 'window of opportunity' for therapeutic intervention, we have conducted a proteomics analysis of CSF from rats at 12 days after cervical spinal cord contusion for changes in 34 cytokines using a multiplex sandwich ELISA microarray. Initial statistical analysis yielded seven proteins (IL-1 $\alpha$, Leptin, B7-2/CD86, GM-CSF, IL-1 $\beta$, matrix metalloproteinase-8 (MMP-8), and tissue inhibitor of metalloproteinase-1 (TIMP-1)), the levels of which were all increased in the CSF from injured animals compared with normal uninjured controls. The FDR was controlled (limit type I errors) by adjusting the $P$-values using both Benjamini-Hochburg and Bonferroni methods (although we chose to focus our analysis on Benjamini-Hochburg adjustments because this method generally allows more potential hits to reach significance). To account for technical replicates, as well as variation in regional signal intensity within the array, a linear mixed model was applied, which showed only MMP-8 was significantly elevated in injured CSF, a finding subsequently confirmed by western blot.

\section{Methods}

\section{Spinal cord injury model}

Animal care and experimental protocols were carried out according to the guidelines of the National Institutes of Health as well as the policies of our Institutional Animal Care and Use Committee at the University of Colorado Anschutz Medical Campus. Female Sprague-Dawley rats (Harlan Laboratories, Denver, CO, USA) aged between 3 months and 5 months were used. Animals were anesthetized with an intraperitoneal injection of ketamine and xylazine, shaved and placed in a stereotactic device for small rodent surgery (David Kopf Instruments, Tujunga, CA, USA). Lubricating eye gel was applied to prevent drying under anesthesia. After cleaning the surgical site, a midline incision was made extending from the shoulder blades to the base of the skull. Another midline incision at the origin of the trapezius muscles was made, and the paraspinal muscles were scraped from the spinous processes and lamina of $\mathrm{C} 2$ to $\mathrm{C} 6$ with a small scalpel blade, exposing the spinal column. Hemostasis was achieved using gauze with light pressure. Once the spinal column was exposed, the posterior longitudinal ligament was cut above and below $\mathrm{C} 4$. Rongeurs were used to perform a $\mathrm{C} 4$ laminectomy, keeping the dura intact.

An Infinite Horizon impactor (Precision Systems and Instrumentation, Fairfax, VA, USA) with a $1.7 \mathrm{~mm}$ tip was used to create a unilateral dorsolateral funiculus contusion of the cervical cord. After laminectomy the animal was secured into the impactor by clamping the vertebral bodies of $\mathrm{C} 3$ and $\mathrm{C} 5$ using Adson stabilizing forceps. Contusion was made by dropping a weight with a preset force of $75 \mathrm{kdynes}$ on the lateral aspect of the cord at the C4 level with a dwell time set at zero. Both displacement and force were recorded to minimize injury variation. The animal was then returned to the stereotactic device. The trapezius muscles were approximated using simple interrupted stitches (4-0 size vicryl suture). Skin was closed with surgical staples.

\section{Cerebrospinal fluid collection}

Animals were again anesthetized at 12 days following contusion and were placed in the stereotactic device. The old incision was extended rostrally between the ears

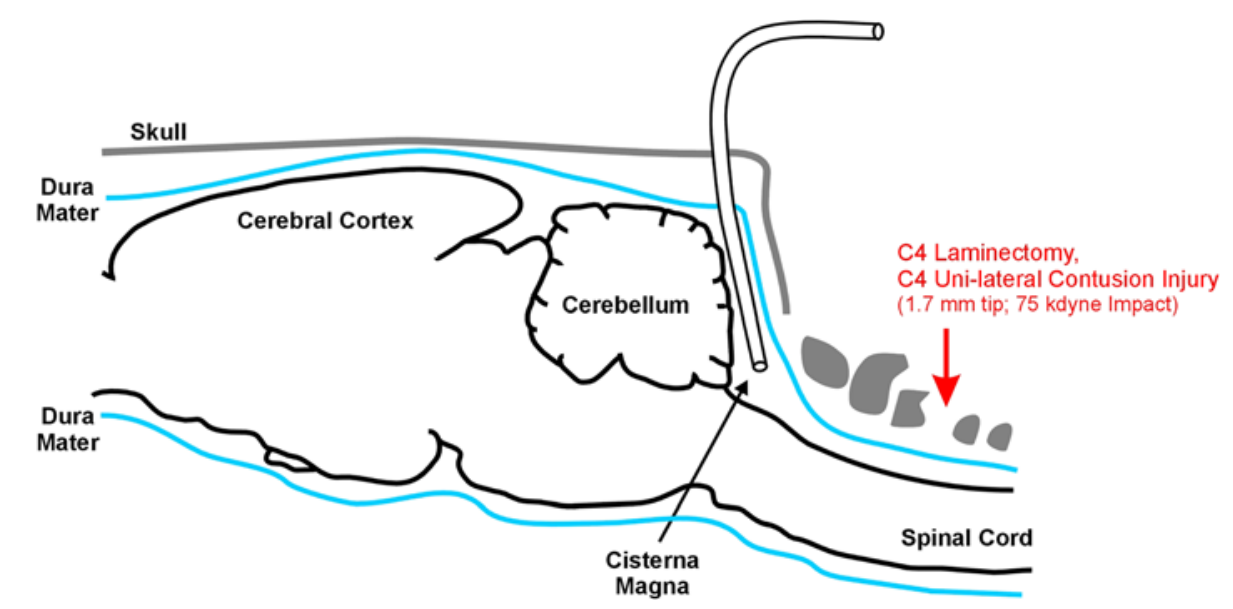

Figure 1 Schematic of contusion injury and cannula placement for CSF extraction. The cannula was guided to a depth of $7 \mathrm{~mm}$ to access the cisterna magna below the cerebellum. 
to expose the occipital bone of the skull. Subcutaneous tissue was scraped off the scull with a scalpel, and a small burr hole (from $1 \mathrm{~mm}$ to $2 \mathrm{~mm}$ diameter) was drilled through the occipital bone, exposing the meninges. A slit was then made in the dura and a small diameter cannula (order number 7741, Durect Corp., Cupertino, CA, USA) was carefully guided under the dura (in a caudal direction) along the internal surface of the occipital bone and into the cisterna magna (depth of $7 \mathrm{~mm}$ ). A syringe was used to draw approximately 200 $\mu \mathrm{L}$ of CSF from each animal, making sure the sample was not bloody. All grossly bloody taps were excluded from the analysis (three in total). Protease inhibitor cocktail (Sigma-Aldrich, St. Louis, MO, USA) was quickly added and samples were placed on dry ice. Samples were stored at $-80^{\circ} \mathrm{C}$ until further use. Animals were then sacrificed by intrahepatic injection of ketamine and xylazine. Control CSF was obtained from age-matched animals (no contusion) in a similar fashion. Figure 1 shows a schematic of injury location and cannula placement for CSF extraction.

\section{Cerebrospinal fluid analysis}

Samples were removed from $-80^{\circ} \mathrm{C}$, thawed on ice, and briefly centrifuged. CSF from injured animals was compared with control CSF $(n=4)$ using the 34 cytokine preconfigured sandwich ELISA Rat Cytokine Array G2 (RayBiotech, Norcross, GA, USA). To reduce batch variability $100 \mu \mathrm{L}$ of each CSF sample (eight total) occupied a single array such that only one chip was used for the experiment. CSF samples were not diluted. The chip was read using a GenePix 4000B Microarray Scanner (Molecular Devices, Sunnyvale, CA, USA). Of note, the array was arranged such that each antibody was spotted twice, creating two technical replicates per protein of interest. Any remaining CSF was aliquoted and placed back at $-80^{\circ} \mathrm{C}$. Specific protocol details can be found at the website of RayBiotech Inc [28]. (http://www.raybiotech.com). A schematic of the chip format and image of representative control and injured arrays in the Cy3 channel are shown in Figure 2. Layout of spotted primary antibodies is shown in Table 1 (adapted from RayBiotech Inc.).

\section{Statistical analysis}

Analysis of the data was by the following steps. The dataset was read into $R$ [29] and each of the 96 probe sets (includes cytokines, positive, and negative controls) were tested for significance. A two sample, equal variance $t$-test was used to test the hypothesis $\mathrm{H} 0: \mu 1=\mu 2$ versus $H 1: \mu 1 \neq \mu 2$ where $\mu 1$ is the mean response for the injured rats and $\mu 2$ is the mean response for the non-injured rats. The response in this case is the difference between the median foreground signal and the

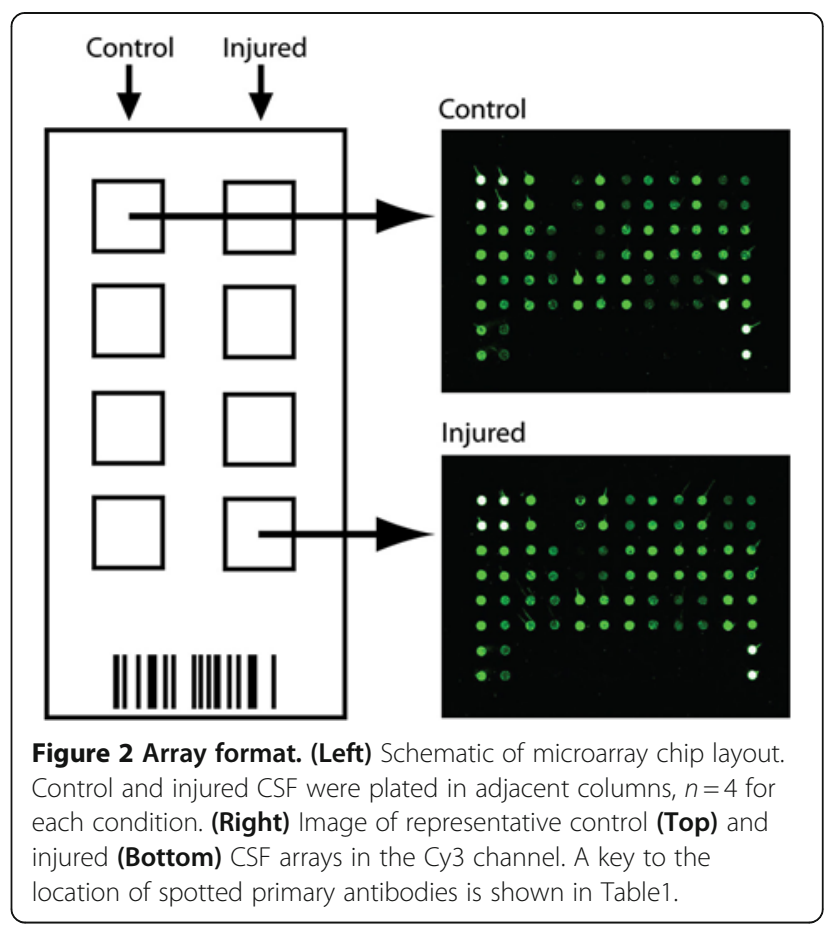

median background signal on the Cy3 channel. Raw $P$-values for each of the 96 probe sets were recorded. Bonferroni and Benjamini-Hochburg multiple testing corrections were used to adjust $P$-values. Accounting for the technical replicates was done using linear mixed models and done in $R$ via the nlme package [30].

\section{Western blot analysis \\ MMP-8 western blot}

The same CSF samples that had been used for microarray were removed from $-80^{\circ} \mathrm{C}$ and thawed on ice. Samples (30 $\mu \mathrm{L}$ each) from two injured and two control animals were run on a $12.5 \%$ polyacrylamide gel (BioRad Life Science, Hercules, CA, USA), and transferred overnight onto PVDF. Westerns were run in duplicate and probed with a polyclonal antibody to MMP-8 (Abcam, Cambridge, MA, USA). Blots were also probed for Transferrin (Santa Cruz Biotechnology, Santa Cruz, CA, USA), previously shown to be unaffected by SCI [27], as a loading control. Rat lung lysate was loaded as a positive control for MMP-8.

\section{TIMP-1 western blot}

CSF from injured and control animals (samples not included in multiplex analysis) were in the same way as MMP-8 western blots (above). Lysate from $293 \mathrm{~T}$ cells over-expressing TIMP-1 (Santa Cruz) and rat whole brain lysate (Abcam) were used for positive controls. The blot was cut and probed separately for transferrin and TIMP-1 (Santa Cruz Biotechnology). 
Table 1 Primary antibody key

\begin{tabular}{|c|c|c|c|c|c|c|c|c|c|c|c|}
\hline Pos & Pos & Pos & $\mathrm{Neg}$ & Act $A$ & Agrin & B7-2/CD86 & $\beta$-NGF & CINC-1 & CINC-2a & CINC-3 & CNTF \\
\hline Pos & Pos & Pos & Neg & Act $\mathrm{A}$ & Agrin & B7-2/CD86 & $\beta$-NGF & CINC-1 & CINC-2a & CINC-3 & CNTF \\
\hline Fas-L & Fract & GM-CSF & ICAM-1 & IFN- $\boldsymbol{\gamma}$ & IL-1a & IL-1 $\beta$ & IL-1 R6 & IL-2 & IL-4 & IL-6 & IL-10 \\
\hline Fas-L & Fract & GM-CSF & ICAM-1 & IFN- $\boldsymbol{\gamma}$ & IL-1a & IL-1 $\beta$ & IL-1 R6 & IL-2 & IL-4 & IL-6 & IL-10 \\
\hline IL-13 & Leptin & LIX & L-Sel & MCP-1 & MIP-3a & MMP-8 & PDGF-AA & PRL- $R$ & RAGE & TK-1 & TIMP-1 \\
\hline IL-13 & Leptin & LIX & L-Sel & MCP-1 & MIP-3a & MMP-8 & PDGF-AA & PRL-R & RAGE & TK-1 & TIMP-1 \\
\hline TNF- $a$ & VEGF & Neg & Neg & Neg & Neg & $\mathrm{Neg}$ & Neg & Neg & Neg & Neg & Neg \\
\hline TNF-a & VEGF & Neg & Neg & Neg & Neg & Neg & Neg & Neg & Neg & Neg & Pos \\
\hline
\end{tabular}

Antibody layout for each array on microarray chip AAR-CYT-G2 (image adapted from RayBiotech). Each primary antibody is spotted twice per array; eight arrays are spotted per chip. Positive and negative controls determine signal variation between arrays.

\section{Results}

\section{Microarray data}

Out of 96 probe sets tested (48 spots with replicates), seven proteins showed a statistically significant difference in median signal intensity (background subtracted) in at least one technical replicate (the response). Proteins with significant results at the $5 \%$ level are reported in Table 2 . Responses from both technical replicates are shown for comparison along with the corresponding raw and adjusted $P$-values. IL-1 $\alpha$, Leptin, B7-2/CD86, GM-CSF, IL-1 $\beta$, MMP-8, and TIMP-1 were all increased in CSF of injured animals compared with controls. Of these results, only IL- $1 \alpha$, MMP-8, and TIMP-1 showed significant increases across both replicate sets after BenjaminiHochburg adjustment. As expected, there were no statistically significant differences among control datasets (data not shown). Bonferroni adjusted $P$-values are shown for comparison.

\section{Microarray data}

To account for technical replicates, as well as regional signal variability across the array as a whole, a linear mixed model was applied to the data. Table 3 displays the full table of results from the mixed model. The value displayed is the difference between the average of median signal intensity minus the median background intensity (across all technical replicates) for injured and control animals. Positive values indicate an increase in protein in injured animals, and negative values indicate a decrease. Results found to have statistically different signals between the injured and non-injured animals based on raw $P$-values are highlighted. In this model, only MMP-8 and Thymus Chemokine- 1 showed statistically significant changes based on raw P-values. MMP-8 was elevated in injured animals: the same conclusion as from the probe set level tests (simple model). Thymus Chemokine-1 did not reach significance in the simple

Table 2 Simple model: two sample equal variance $t$-tests

\begin{tabular}{llllll}
\hline ID & Response Injured & Response non-Injured & Raw $\boldsymbol{P}$-value & BH adj. $\boldsymbol{P}$-value & Bonferroni adj. $\boldsymbol{P}$-value \\
\hline IL-1a & 418.75 & 349.25 & 0.0019 & 0.0287 & 0.3674 \\
\hline IL-1a & 438.75 & 368.00 & 0.0024 & 0.0287 & 1.0000 \\
\hline Leptin & 839.75 & 733.50 & 0.0005 & 0.0156 & 0.1149 \\
\hline Leptin & 838.00 & 763.00 & 0.2189 & 0.3649 & 1.0000 \\
\hline B7-2/CD86 & 504.25 & 386.50 & 0.0115 & 0.0927 & 0.1818 \\
\hline B7-2/CD86 & 533.75 & 413.50 & 0.0038 & 0.0367 & 0.2296 \\
\hline GM-CSF & 1223.25 & 1012.00 & 0.0132 & 0.0932 & 0.2194 \\
\hline GM-CSF & 1204.75 & 1002.50 & 0.0012 & 0.0287 & 1.0000 \\
\hline IL-1 $\beta$ & 941.50 & 823.75 & 0.0200 & 0.1121 & 0.0468 \\
\hline IL-1 3 & 992.25 & 818.00 & 0.0023 & 0.0287 & 1.0000 \\
\hline MMP-8 & 36424.75 & 3360.25 & 0.0003 & 0.0123 & 0.0205 \\
\hline MMP-8 & 36255.50 & 3567.75 & 0.0002 & 0.0123 & 0.0246 \\
\hline TIMP-1 & 4181.50 & 1720.50 & 0.0028 & 0.0304 & 0.2153 \\
\hline TIMP-1 & 4099.50 & 1688.25 & 0.0022 & 0.0287 & 0.2732
\end{tabular}

Mean response by injury status with raw and adjusted $P$-values for significance between injured and non-injured animals. Response is the difference between the median foreground signal and the median background signal on the Cy3 channel. Raw $P$-values are shown with Benjamini-Hochburg adjustments. Bonferroni adjusted $P$-values are shown for comparison. Proteins in which changes in both technical replicates were significant at the $5 \%$ level (BH adjusted $P$-value) are italicized. 
Table 3 Linear mixed model results

\begin{tabular}{|c|c|c|c|}
\hline ID & Response difference & Raw $P$-value & BH adjusted $P$-value \\
\hline MMP-8 & 32856.83 & 0.0000 & 0.0000 \\
\hline Thymus Chemokine-1 & -8426.12 & 0.0163 & 0.3094 \\
\hline$\overline{M C P}-1$ & 4878.50 & 0.1624 & 0.9978 \\
\hline TIMP-1 & 2416.63 & 0.4882 & 0.9978 \\
\hline IL-13 & -1482.87 & 0.6705 & 0.9978 \\
\hline Agrin & 658.13 & 0.8502 & 0.9978 \\
\hline TNF-a & 383.13 & 0.9124 & 0.9978 \\
\hline Fractalkine & 214.00 & 0.9510 & 0.9978 \\
\hline PDGF-AA & 196.13 & 0.9551 & 0.9978 \\
\hline GM-CSF & 187.25 & 0.9571 & 0.9978 \\
\hline$\overline{I L}-4$ & 175.63 & 0.9598 & 0.9978 \\
\hline$\overline{\mathrm{IL}-2}$ & 171.75 & 0.9607 & 0.9978 \\
\hline VEGF & 160.25 & 0.9633 & 0.9978 \\
\hline ICAM-1 & 152.88 & 0.9650 & 0.9978 \\
\hline Fas ligand & -138.75 & 0.9682 & 0.9978 \\
\hline$I L-1 \beta$ & 126.50 & 0.9710 & 0.9978 \\
\hline LIX & 124.75 & 0.9714 & 0.9978 \\
\hline$\beta-N G F$ & 120.75 & 0.9724 & 0.9978 \\
\hline L-selectin & 118.38 & 0.9729 & 0.9978 \\
\hline MIP-3a & 109.38 & 0.9750 & 0.9978 \\
\hline IL-10 & 100.13 & 0.9771 & 0.9978 \\
\hline B7-2/CD86 & 99.50 & 0.9772 & 0.9978 \\
\hline IL-1 R6 & 97.63 & 0.9776 & 0.9978 \\
\hline Leptin & 71.13 & 0.9837 & 0.9978 \\
\hline IL-6 & 58.38 & 0.9866 & 0.9978 \\
\hline IL-1a & 50.63 & 0.9884 & 0.9978 \\
\hline RAGE & 30.00 & 0.9931 & 0.9978 \\
\hline Activin A & 19.50 & 0.9939 & 0.9978 \\
\hline CINC-2a & -26.37 & 0.9940 & 0.9978 \\
\hline Prolactin- $R$ & 24.13 & 0.9945 & 0.9978 \\
\hline CINC-3 & 20.88 & 0.9952 & 0.9978 \\
\hline CNTF & 16.63 & 0.9962 & 0.9978 \\
\hline CINC-1 & 14.63 & 0.9967 & 0.9978 \\
\hline IFN- $\gamma$ & 9.63 & 0.9978 & 0.9978 \\
\hline
\end{tabular}

$\mathrm{BH}$, Benjamini-Hochburg; MMP, matrix metalloproteinase; TIMP-1, tissue inhibitor of metalloproteinase-1.

Full table of results from mixed model sorted by raw $P$-value. The differences in means between injured and non-injured animals are displayed in the second column. Proteins with changes significant at the $5 \%$ level (BH adjusted $P$-values) are italicized.

model, but showed a statistically significant decrease in the linear mixed model based on the raw P-value; a result that did not hold up after Benjamini-Hochburg adjustment. This discrepancy is the result of significant variability within the Thymus Chemokine-1 dataset combined with large differences in the magnitude of signal between injured and control animals. After BenjaminiHochburg correction of mixed model data, MMP-8 was the only cytokine with a significant change between injured and non-injured animals $(P<0.0001)$. Figure 3 shows all median response data $(n=8)$ from the MMP-8 and TIMP-1 datasets in box plot format.

\section{Western blot analysis}

To confirm that microarray samples contained a protein with the correct molecular weight for MMP-8, CSF from injured and control animals were run and probed for MMP-8. A probe for Transferrin was used as a loading control. Westerns for both MMP-8 and Transferrin were aligned to create Figure 4(a). One representative set of 


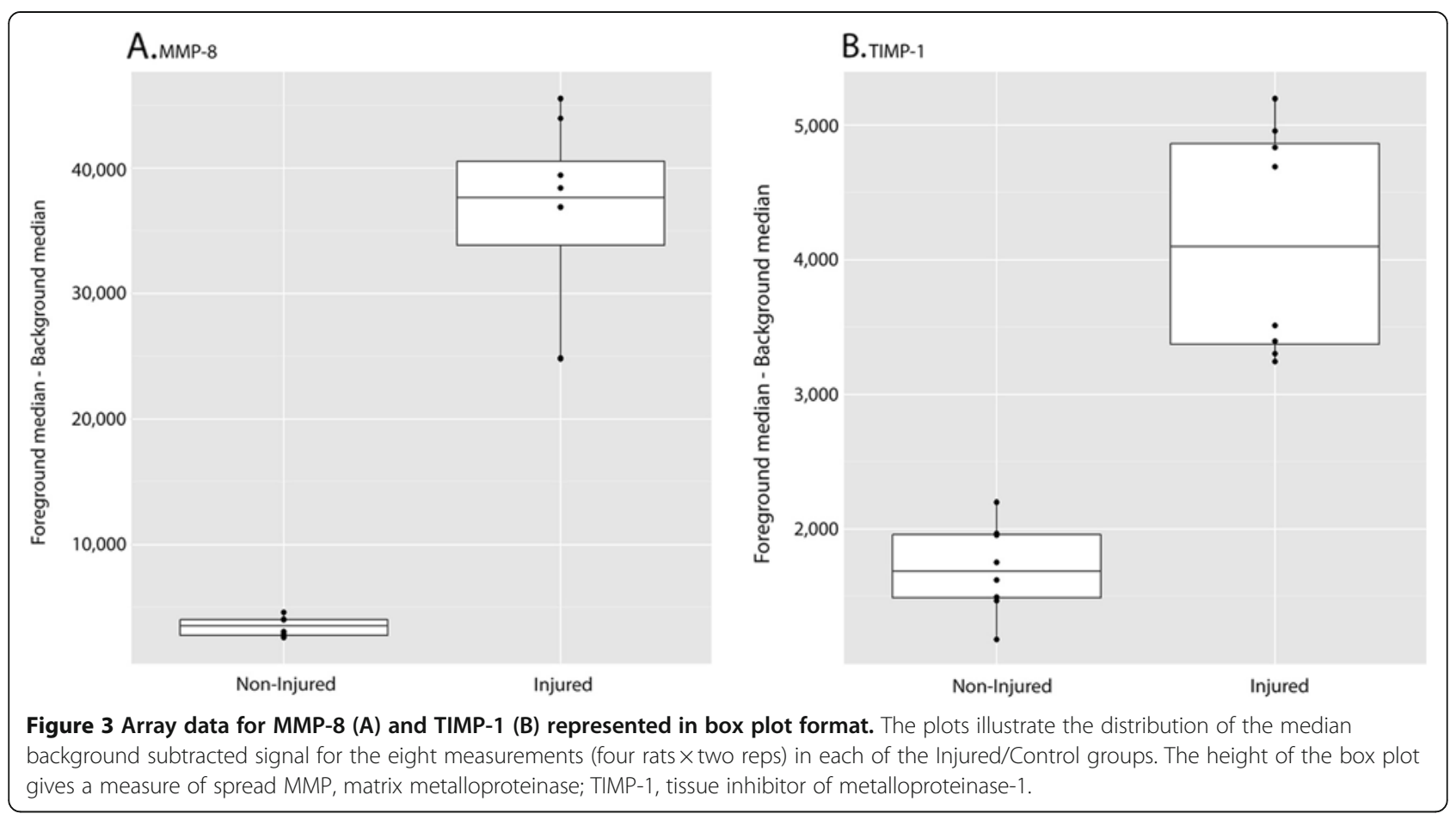

data is shown. Western blot for MMP-8 shows a band at approximately $53 \mathrm{kD}$ (predicted size for rat MMP-8) in the lung positive control, as well as bands of the same size in each CSF sample. A clear increase in MMP-8 protein was observed in samples from injured animals compared with controls, but more importantly there was no

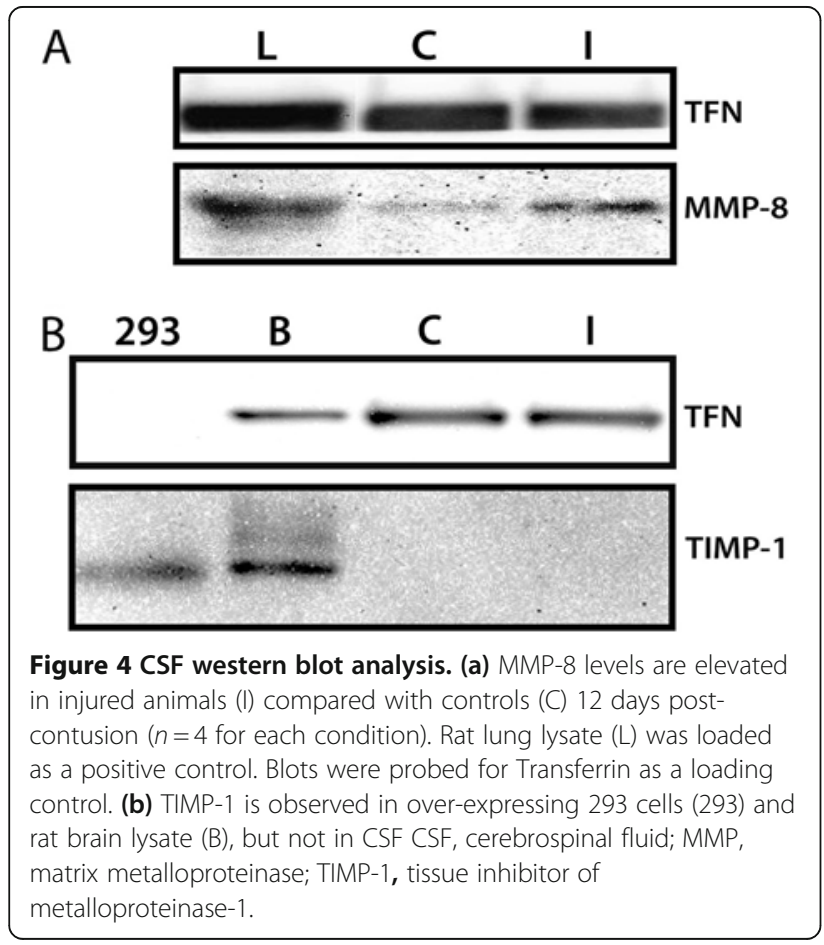

evidence of immune-reactive proteins or breakdown products. The Transferrin blot shows comparable total protein concentrations in each sample as indicated by relative band size/intensity.

Western blot for TIMP-1 was also performed to test for type II errors in the statistical analysis. Specifically we were interested in testing whether there were hits present in the simple model that did not reach significance in the mixed model. The western blot for TIMP-1 along with Transferrin loading controls are shown in Figure 4(b). Bands at approximately $25 \mathrm{kD}$ are seen in positive controls (cells overexpressing TIMP-1 and brain extracts) but not in CSF samples. This result is not unexpected given the relatively weak signal intensity for TIMP-1 in the multiplex array compared with MMP-8, which indicated lower protein concentrations (or weaker antigen-antibody affinity) that were likely below the limit of detection by our western blot system.

\section{Discussion}

The primary objective of this study was to characterize the inflammatory profile present in CSF at a subacute time point in a clinically relevant rodent model of traumatic SCI. Our other aim was to test a microarray proteomics platform specifically for this application. Studying inflammation at 12 days post-SCI is highly relevant in that it falls within a clinical 'window of opportunity' where patients have been stabilized and are generally more receptive to therapy. We also agree with the SCI field in general that CSF is an important source of 
potential biomarkers given that it is safe to obtain, readily available, and in close proximity to neuronal tissue of interest. Using small volumes of CSF we were able to study 34 cytokines/growth factors simultaneously, detecting significant changes in seven inflammatory markers. After applying statistical models designed for multiplex testing, we also found robust increases in MMP-8 within CSF, which to our knowledge represents a novel subacute phase biomarker for SCI.

As with any new technology, comparison of results to previous data is crucial in assessing the reliability of the new system. It is generally accepted that a multiphasic inflammatory response occurs after SCI: an early phase peaking at 24 hours and extending from 9 to 10 days, and a later phase between 14 and 180 days post-injury [31]. Studies of inflammation in the setting of SCI have primarily focused their analysis on this early phase between 0 and 72 hours post-injury when neuroprotective strategies are most effective, making comparisons to this study somewhat difficult. Changes in mRNA from rat cord lysate observed by Streit et al. (IL-1 $\beta$, TNF- $\alpha$, IL-6, and $\mathrm{M}-\mathrm{CSF}$ ) were transient, returning to normal levels by 24 hours post-injury, and remaining at these levels for the remainder of their analysis (10 days) [24]. In this study, TGF- $\beta$ levels started to rise at 24 hours, peaked at 7 days, and remained elevated at 10 days; TGF- $\beta$ was not included in our analysis. The study by Wang et al. showed elevated IL-1 $\beta$ protein in CSF at one hour [25]. Their result differed from Streit et al. in that protein levels remained elevated at 7 days, but fits nicely with our IL-1 $\beta$ simple model result. Although changes in IL$1 \beta$ did not hold up to mixed model analysis, it is quite possible that this result is real, and that we are observing a lull in protein concentration between early- and latephase inflammation. Further analysis is needed to confirm this result. Yang et al. showed elevated levels of IL$1 \beta$, IL- 6 , and TNF- $\alpha$ by western blot from cord lysates, a result that peaked at 6 hours, returned to normal by 24 hours, and was dependent on injury severity [6]. IL-6 and TNF- $\alpha$ levels in our study, although elevated in injured animals, did not reach statistical significance. Finally, the human CSF study by Kwon and colleagues did not show any changes in IL-1 $\beta$ or GM-CSF protein over a 5-day period [26] (both elevated in our simple model at 12 days), a difference that may be explained by the observation window, and/or cross-species differences in the inflammatory response.

MMPs and their endogenous inhibitors (TIMPs) have been studied extensively in the setting of SCI. de Castro et al. showed increases in gelatinases MMP-9 and MMP2 at 12 to14 hours, and 5 days respectively in injured rat spinal cord by zymography. They suggest (using neutralizing antibody studies) that these MMPs are released by infiltrating neutrophils responding to inflammation [32].
Wells et al. performed a comprehensive MMP/TIMP mRNA profile in an acute murine model of SCI. They showed an increase in MMP-3, 7, 10, 11, 19, and 20 at 24 hours post-SCI, and elevated levels of MMP-2, 12, and 13 , but not MMP- 8 at 5 days, colocalizing MMP-12 mRNA to Iba1 positive macrophages in the lesion epicenter [33].Using microarray proteomics, we present a new finding, an increase in MMP-8 protein, also known as neutrophil collagenase or collagenase-2, in CSF at 12 days after SCI in rats. This result is consistent with findings of Veeravalli et al. who showed an increase in MMP-8 mRNA following SCI between 3 and 21 days post-injury in mouse cord lysate samples [34]. Increases in TGF- $\beta$ mRNA 10 days post-SCI reported by Streit et al. [24] may in part explain the elevated levels of MMP-8 in our study, as TGF- $\beta$ is a known inducer of other collagenases like MMP-13, through the Smad3 pathway [35], as well as MMP-2, and MMP-9 though ERK-1/2, and p38 MAPK [36]. In the Streit study [24], TGF- $\beta$ levels started to rise at 24 hours, peaked at 7 days, and remained elevated at 10 days; TGF- $\beta$ however was not included in our analysis. Our finding also correlates with data from the stroke field, where Cuadrado and colleagues reported upregulation of MMP-8 protein (and other MMPs) in ischemic brain tissue [37]. Finally, the fact that increases in CSF MMP-8 protein levels were not reported by Lubieniecka et al.[27], despite the use of sensitive mass spectrometry analysis at time points ranging from 12 to 72 hours after injury, indicates that our observed increase in MMP-8 protein in CSF represents a novel subacute-phase SCI biomarker. Increases in a variety of MMPs within the spinal cord following SCI are thought to significantly contribute to further tissue damage particularly at acute time points after SCI. At present, however, relatively little is known about CSF levels of specific MMP mRNAs and proteins or their impact on tissue damage at subacute time points after SCI. Elevated levels of MMP-12 mRNA in spinal cord injured tissue observed by Wells and colleagues [33] have been shown to correlate with disruption of the BSCB, however this study did not extend beyond 5 days post-SCI. Whether the significant increase in MMP-8 protein we have observed in the CSF contributes to BSCB disruption and other mechanisms of tissue damage post-SCI requires further investigation beyond the scope of the present study.

Comparing MMP-8 and TIMP-1 signals in both microarray statistical models illustrates the importance of data interpretation in multiplex testing. MMP-8, by far the greatest protein change detected, was significant across both models, suggesting the ability of the array and mixed statistical model combination to correctly identify true changes in the dataset. We would like to reiterate the importance of methods like western blot in 
verifying protein size when using an ELISA-based system because other immunoreactive proteins may disrupt the analysis.

TIMP-1, by contrast, only reached statistical significance in our simple model. Lack of significance in the linear mixed model indicates that this result is more likely to be a type 1 error than a true positive. Western blot was performed to test this hypothesis. Although it is possible that our inability to detect TIMP by western blot reflects the ability of the mixed model to control the FDR, it is also possible that TIMP-1 is truly elevated following $\mathrm{SCI}$ and, as discussed above, the microarray was just more sensitive at identifying biologically significant changes in this molecule.

Clearly, uncertainty exists when interpreting the results of multiplex testing. Different statistical treatment of the data gives us clues, but follow-up testing is often required by conventional methods. This is especially true when using the Benjamini-Hochburg correction, accepting a certain number of false positives per assay. However, there are ways, within the confines of the multiplex format, to reduce uncertainty. One obvious option would be to increase the sample size of each dataset. If we were to run two chips instead of one would we not increase our power to detect differences in the data? This brings up the concept of batch variability: the observation of significant variation between batches (chips in our experiment). This variability commonly offsets any statistical 'gain' from the corresponding increase in sample size. A better alternative would be to run sequential arrays, one for screening, as presented here, and another 'confirmation array', where only hits from the screening array are spotted, in greater numbers. This sequential approach, although more expensive and time consuming, avoids the problem of batch variation, and prevents controls for the FDR from making costly type II errors.

\section{Conclusions}

To our knowledge our study is the first to demonstrate significant increases in MMP-8 protein in CSF after traumatic injury to the spinal cord. We propose that MMP-8 is a novel subacute-phase CSF biomarker for SCI. In addition, our study supports the use of sandwich microarray proteomics as a valid approach for studying CSF in the setting of SCI in rats, and potentially human SCI patients. This assay reliably detects low levels of CSF protein, and has several advantages over conventional methods including quick and easy protocol, highthroughput format, and use of relatively small amounts of precious sample. Disadvantages include relative cost compared with traditional methods, and the need to confirm hits (either with repeat microarray analysis or other assays). Moreover our study stresses the importance of controlling the FDR given the multiplex format. In summary, this assay has the potential to detect new target molecules, characterize complex time-dependent pathophysiologic responses to SCI (like inflammation) and, as more therapies reach the clinic, identify biomarkers with which to assess injury severity and grade response to treatment.

\section{Abbreviations}

ASIA: American Spinal Injury Association; BH: Benjamini-Hochburg multiple testing correction; BSCB: blood-spinal cord barrier; CNS: central nervous system; CSF: cerebrospinal fluid; ELISA: enzyme-linked immunosorbent assay; FDR: false discovery rate; IL: interleukin; MMP: matrix metalloproteinase; mRNA: messenger RNA; PCR: polymerase chain reaction; SCl: spinal cord injury; TIMP: tissue inhibitor of metalloproteinase; TNF: tumor necrosis factor.

\section{Competing interests}

The authors declare that they have no competing interests.

\section{Authors' contributions}

$\mathrm{ML}, \mathrm{KM}$, and SD designed the study. KM conducted all animal injuries and KM and ML performed CSF extractions. ML and KJ performed the microarray and western blot analysis. PD performed all statistical analysis. Manuscript was written by ML, PD, KM and SD. All authors have read and approved the final manuscript.

\section{Acknowledgements}

Funding for the study was provided in part by the Lone Star Paralysis Foundation, Lloyd and Floyd Holman, the David Van Wagener SCI Fund, and the CareCure SCl community.

We acknowledge Martha Miles and Sean Carriedo of Molecular Devices Corp. who provided technical assistance.

\section{Author details}

'Department of Neurosurgery, University of Colorado School of Medicine, Building RC-1 North, Room P18-9400, 12800 E 19th Avenue, Aurora, CO 80045, USA. ${ }^{2}$ Colorado Biostatistics Consortium, Department of Biostatistics and Informatics, University of Colorado Denver, 12477 E 19th Avenue, Room 102, Aurora, CO 80045, USA.

Received: 23 November 2011 Accepted: 4 May 2012

Published: 11 June 2012

\section{References}

1. National Spinal Cord Injury Statistical Center: Spinal cord injury: facts and figures at a glance. J Spinal Cord Med 2005, 28:379-380.

2. Miniño AM: Death in the United States, 2009. NCHS data brief, no. 64. Hyattsville, MD: National Center for Health Statistics; 2011. http://www.cdc. gov/nchs/data/databriefs/db64.pdf.

3. van den Berg ME, Castellote JM, de Pedro-Cuesta J, Mahillo-Fernandez I: Survival after spinal cord injury: a systematic review. J Neurotrauma 2010, 27:1517-1528.

4. Fleming JC, Norenberg MD, Ramsay DA, Dekaban GA, Marcillo AE, Saenz AD, Pasquale-Styles M, Dietrich WD, Weaver LC: The cellular inflammatory response in human spinal cords after injury. Brain: J Neurol 2006, 129:3249-3269.

5. Leal-Filho MB: Spinal cord injury: from inflammation to glial scar. Surg Neurol Int 2011, 2:112.

6. Yang L, Jones NR, Blumbergs PC, Van Den Heuvel C, Moore EJ, Manavis J, Sarvestani GT, Ghabriel MN: Severity-dependent expression of proinflammatory cytokines in traumatic spinal cord injury in the rat. J Clin Neurosci 2005, 12:276-284.

7. Blight AR: Macrophages and inflammatory damage in spinal cord injury. $J$ Neurotrauma 1992, 9(Suppl. 1):S83-S91.

8. Pruss H, Kopp MA, Brommer B, Gatzemeier N, Laginha I, Dirnagl U, Schwab JM: Non-Resolving Aspects of Acute Inflammation after Spinal Cord Injury (SCI): Indices and Resolution Plateau. Brain Pathol 2011, 21:652-660.

9. Cohen DM, Patel CB, Ahobila-Vajjula P, Sundberg LM, Chacko T, Liu SJ, Narayana PA: Blood-spinal cord barrier permeability in experimental spinal cord injury: dynamic contrast-enhanced MRI. NMR Biomed 2009, 22:332-341. 
10. Ankeny DP, Popovich PG: Mechanisms and implications of adaptive immune responses after traumatic spinal cord injury. Neuroscience 2009, 158:1112-1121.

11. Fehlings MG, Hawryluk GW: Scarring after spinal cord injury. J Neurosurg Spine 2010, 13:165-167. discussion 167-168.

12. Silver J, Miller JH: Regeneration beyond the glial scar. Nat Rev Neurosci 2004, 5:146-156

13. Gensel JC, Donnelly DJ, Popovich PG: Spinal cord injury therapies in humans: an overview of current clinical trials and their potential effects on intrinsic CNS macrophages. Exp Opin Therapeutic Targets 2011 , 15:505-518.

14. Kwon BK, Casha S, Hurlbert RJ, Yong W: Inflammatory and structural biomarkers in acute traumatic spinal cord injury. Clin Chem Lab Med: CCLM/FESCC 2011, 49:425-433.

15. Reiber $\mathrm{H}$ : Cerebrospinal fluid-physiology, analysis and interpretation of protein patterns for diagnosis of neurological diseases. Mult Scler 1998, 4:99-107.

16. Lee N, Wong CK, Chan PK, Chan MC, Wong RY, Lun SW, Ngai KL, Lui GC, Wong BC, Lee SK, Choi KW, Hui DSC: Cytokine response patterns in severe pandemic $2009 \mathrm{H} 1 \mathrm{~N} 1$ and seasonal influenza among hospitalized adults. PLoS One 2011, 6:e26050.

17. Mizutani T, Akasaka R, Tomita K, Chiba T: Serial changes of cytokines in Crohn's disease treated with infliximab. Hepatogastroenterol 2011, 58(110111):1523-1526

18. Tefferi A, Vaidya R, Caramazza D, Finke C, Lasho T, Pardanani A: Circulating interleukin (IL)-8, IL-2R, IL-12, and IL-15 levels are independently prognostic in primary myelofibrosis: a comprehensive cytokine profiling study. J Clin Oncol 2011, 29:1356-1363.

19. Nielsen $U B$, Geierstanger $B H$ : Multiplexed sandwich assays in microarray format. J Immunol Methods 2004, 290:107-120.

20. Pang S, Smith J, Onley D, Reeve J, Walker M, Foy C: A comparability study of the emerging protein array platforms with established ELISA procedures. J Immunol Methods 2005, 302:1-12.

21. Benjamini $Y$, Hochburg Y: Controlling the false discovery rate: a practical and powerful approach to multiple testing. J Royal Stat Soc Series B (Methodol) 1995, 57:289-300.

22. Hsueh HM, Chen JJ, Kodell RL: Comparison of methods for estimating the number of true null hypotheses in multiplicity testing. J Biopharm Stat 2003, 13:675-689.

23. Zhao M, Wang X, Nolte D: Mass-transport limitations in spot-based microarrays. Biomed Opt Express 2010, 1:983-997.

24. Streit WJ, Semple-Rowland SL, Hurley SD, Miller RC, Popovich PG, Stokes BT: Cytokine mRNA profiles in contused spinal cord and axotomized facial nucleus suggest a beneficial role for inflammation and gliosis. Exp Neurol 1998, 152:74-87

25. Wang CX, Olschowka JA, Wrathall JR: Increase of interleukin-1 beta mRNA and protein in the spinal cord following experimental traumatic injury in the rat. Brain Res 1997, 759:190-196.

26. Kwon BK, Stammers AM, Belanger LM, Bernardo A, Chan D, Bishop CM Slobogean GP, Zhang H, Umedaly H, Giffin M, Street J, Boyd MC, Paquette SJ, Fisher CG, Dvorak MF: Cerebrospinal fluid inflammatory cytokines and biomarkers of injury severity in acute human spinal cord injury. $J$ Neurotrauma 2010, 27:669-682.

27. Lubieniecka JM, Streijger F, Lee JH, Stoynov N, Liu J, Mottus R, Pfeifer T, Kwon BK, Coorssen JR, Foster LJ, Grigliatti TA, Tetzlaff W: Biomarkers for severity of spinal cord injury in the cerebrospinal fluid of rats. PLOS One 2011, 6:e19247.

28. RayBiotech, Inc:, . http://www.raybiotech.com (November 12, 2011).

29. Team RDC: R: A Language and Environment for Statistical Computing. Vienna, Austria: R Foundation for Statistical Computing: 2011.

30. Pinheiro J, Bates D, DebRoy S, Sarkar D, nlme: Linear and Nonlinear Mixed Effects Models. $R$ package version 3.1-102 edition. Vienna, Austria: $R$ Foundation for Statistical Computing; 2011

31. Beck KD, Nguyen HX, Galvan MD, Salazar DL, Woodruff TM, Anderson AJ: Quantitative analysis of cellular inflammation after traumatic spinal cord injury: evidence for a multiphasic inflammatory response in the acute to chronic environment. Brain 2010, 133:433-447.

32. De Castro RC Jr, Burns CL, MCAdoo DJ, Romanic AM: Metalloproteinase increases in the injured rat spinal cord. NeuroReport 2000, 11:3551-3554
33. Wells JE, Rice TK, Nuttall RK, Edwards DR, Zekki H, Rivest S, Yong WW: An adverse role for matrix metalloproteinase 12 after spinal cord injury in mice. J Neurosci 2003, 23:10107-10115.

34. Veeravalli KK, Dasari VR, Tsung AJ, Dinh DH, Gujrati M, Fassett D, Rao JS: Human umbilical cord blood stem cells upregulate matrix metalloproteinase-2 in rats after spinal cord injury. Neurobiol Dis 2009, 36:200-212

35. Leivonen SK, Chantry A, Hakkinen L, Han J, Kahari VM: Smad3 mediates transforming growth factor-beta-induced collagenase-3 (matrix metalloproteinase-13) expression in human gingival fibroblasts. Evidence for cross-talk between Smad3 and p38 signaling pathways. J Biol Chem 2002, 277:46338-46346.

36. Kim ES, Kim MS, Moon A: TGF-beta-induced upregulation of MMP-2 and MMP-9 depends on p38 MAPK, but not ERK signaling in MCF10A human breast epithelial cells. Int J Oncol 2004, 25:1375-1382

37. Cuadrado E, Rosell A, Penalba A, Slevin M, Alvarez-Sabin J, Ortega-Aznar A, Montaner J: Vascular MMP-9/TIMP-2 and neuronal MMP-10 up-regulation in human brain after stroke: a combined laser microdissection and protein array study. J Proteome Res 2009, 8:3191-3197.

doi:10.1186/1742-2094-9-122

Cite this article as: Light et al:: Multiplex array proteomics detects increased MMP-8 in CSF after spinal cord injury. Journal of Neuroinflammation 2012 9:122.

\section{Submit your next manuscript to BioMed Central and take full advantage of:}

- Convenient online submission

- Thorough peer review

- No space constraints or color figure charges

- Immediate publication on acceptance

- Inclusion in PubMed, CAS, Scopus and Google Scholar

- Research which is freely available for redistribution 